\title{
Crab Larvae Counter Using Image Processing
}

\author{
Furqan Zakiyabarsi ${ }^{\mathrm{a}, *}$, Muhammad Niswar $^{\mathrm{b}}$, Zahir Zainuddin $^{\mathrm{c}}$ \\ ${ }^{a}$ Electrical Engineering Department, Engineering Faculty, Hasanuddin University. Email: thb.zone@gmail.com \\ ${ }^{\text {b}}$ Electrical Engineering Department, Engineering Faculty, Hasanuddin University. Email: mniswar@ gmail.com \\ 'Electrical Engineering Department, Engineering Faculty, Hasanuddin University. Email: zainuddinzahir@ gmail.com
}

\begin{abstract}
Very small larval size, high mortality rate of crab larvae, the absence of an accurate crab larva counter at affordable price, the density of larvae that are not known accurately, the ratio of feed that not in accordance with the number of larvae to damage water quality and cannibalism, water quality cannot be adjusted to the density of distribution, and economically unprofitable both in terms of feed efficiency and buying and selling transactions. By knowing the number of crab larvae accurately the survival rate of crab larvae increas es and is economically beneficial. In this study, larvae counters were designed and developed. This tool uses digital imagery to detect and count crab larvae zoea-1 phase. The accuracy of the crab larva counter is $89,8 \%$.
\end{abstract}

Keywords: Counter application; crab; high mortality; image processing; larval; pattern recognition

\section{Introduction}

The increasing number of population drives the need for better food and nutrition continues to increase. The phenomenon of shifting orientation towards meeting people's food needs that prioritizes safe and healthy food consumption, has directly triggered a high demand for alternative protein needs. In other words, the consumption pattern has shifted from red meat-based food products to white meat-based food products, in this case is seafood, one of the highest demand is crab.

This has driven the market demand for the need for protein-rich consumption of crabs to increase and Indonesia is one of the crab producing countries for domestic and foreign market needs. However, the survival rate from crab larvae to adult crabs is very low, the highest survival rate of zoea to megalopa is $14.12 \pm 031 \%$ [1] and in general crab larvae only have the highest survival rate between $20-50 \%$ [2] has become a serious problem in terms of crab production in order to immediately find a solution in terms of technology implementation. The absence of a tool that is able to accurately calculate crab larvae at an affordable price in an easy way to do is forcing farmers to count traditionally with sampling methods and estimates that are very at risk of increasing mortality in the cultivation process because the level of larval density is not known accurately causing feed ratio and number of larvae are not comparable. The ratio of feed that exceeds the needs of larvae will be polluted, high levels of cannibalism due to lack of feed, and economically the number of larvae that is inaccurate can harm both cultivators with high mortality rates and consumers with the number of larvae purchased are not known accurately and can reduce market trust. Larva counting methods currently carried out by aquaculture practitioners only use the population sampling method and look for the average of several sampling. Even traditional aquaculture practitioners use more estimates in terms of determining the number of crab larvae.

Digital image processing is one of the technologies that can fill this gap. Image processing utilizes digital images with certain techniques to get more information from these images. The increasingly advanced and affordable image processing technology infrastructure makes image processing technology very good and easy to implement and absorb by the public.

Based on this, the author raised the title of the Crab Processing Larvae Using Image Processing to provide information about the number of crab larvae that can be used for aquaculture needs and economic needs in an easy and affordable price.

\section{Methodology}

\subsection{Image Acquisition}

Taking pictures using a smartphone with camera specifications above 8 Mega Pixels that have been used by most Indonesian people, so that the implementation of this research can be well absorbed by the public. This research uses the following instrumentation: 
- Software
a. Mac OS Sierra
b. Anaconda (Python)
c. OpenCV

- Hardware

a. Laptop MacBook Air with Prosesor Core i5 (1.40 $\mathrm{GHz}$ )

b. Solid State Drive 128 GB Intel

c. Memory DDR3 4 GB

d. Monitor 11"

e. Handphone Android Oppo F7, Vivo V9, dan Vivo V15

\subsection{Model Description}

The system design in this study was designed so that this technology is easily absorbed and used by the public. The larvae to be counted are put into special containers that have special lighting, after that using a mobile camera the larvae picture is taken. The results of the images obtained are then processed using image processing methods to get an accurate number of crab larvae.

Image processing method, using an image processing technique as shown in Fig. 2.

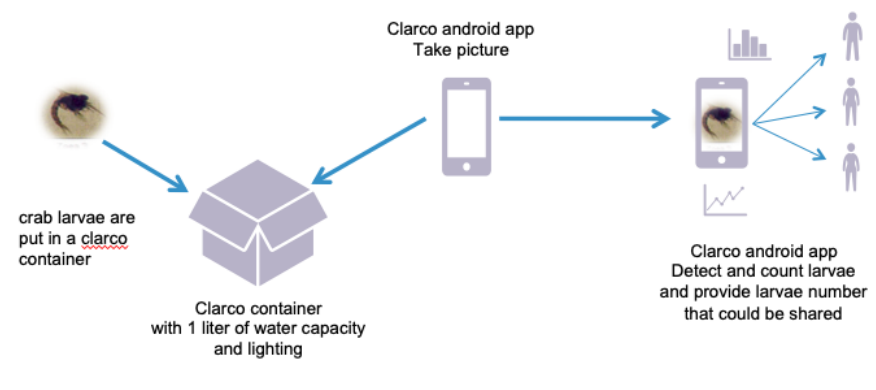

Figure 1. System design

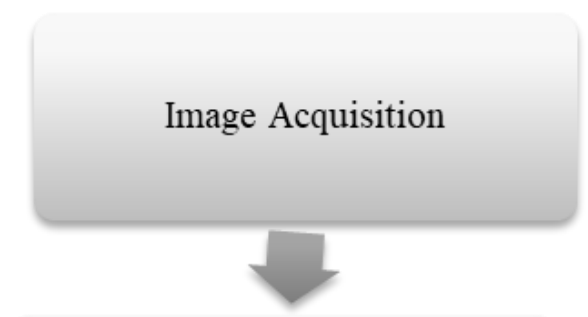

Image Processing Technique

- Image Resize

- Gray-Level Technique

- Segmentation and Detection Technique

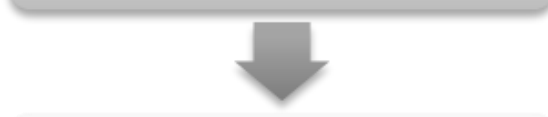

Larval Number Information

Figure 2. Model research methods

\subsection{Image Processing Technique}

Pixels represent picture elements and it is the smallest unit of picture information that is stored as bits to form a digital image. In general, more bits stored per pixel will result in a clearer image due to higher greyscale or color resolution in the image, and the more pixels used to represent an image, the closer the image resembles the original. In imaging science, the analysis, manipulation, storage, and display of pixel information from sources as photographs, drawings, and video refers to image processing. Output of the image processing is an image or a set of characteristics or parameters related to the image [3].

\subsubsection{Image Resize}

The initial step to do is to resize the image size so that the number of pixels is as desired and does not overload memory performance.

\subsubsection{Grey-Level Technique}

Thresholding or gray-level segmentation is an essential concept related to image processing and machine vision. Thresholding is a conversion between a gray-level image and a bi-level image. Bilevel image is a monochrome image only composed of black and white pixels. It should contain the most essential information of the image (i.e., number, position, and shape of objects), but is not comparable with the information offered by the grey-level image. Most of the time pixels with similar grey levels belong to the same object. Therefore, classifying the image by grey-level pixels may reduce and simplify some image processing operations such as pattern recognition, and classification [4].

Thresholding operations select pixels whose component values lie within a specified range. The operation may change the values of either the selected or the unselected pixels. A pixel pattern can be highlighted, for example, by setting all the pixels in the pattern to 0. Pixel maps and lookup tables provide a simple mechanism for thresholding using individual component values. However, pixel maps and lookup tables only allow replacement of one component individually, so lookup table thresholding is trivial only for single component images [5].

All methods so far assumed that the background and object pixels have non-overlapping grey levels. Most of the time in real-life that ideal assumption is not true. Therefore, the selection of a single threshold for an image is not possible in most of the case. However, all that is needed is for the two classes of pixels not to overlap over each of a set of regions that collectively form the image. The first step is to determine how many independent regions form the image and their sizes. For each of them, it is possible to apply some thresholding methods. Therefore, there is not a single threshold value, but one per region. Only one thing to take care of when using regional thresholding method, it is necessary to make sure that either each region contains a sample of both object and background pixels, or that no thresholding is attempted when only a pixel class exists [4]. 


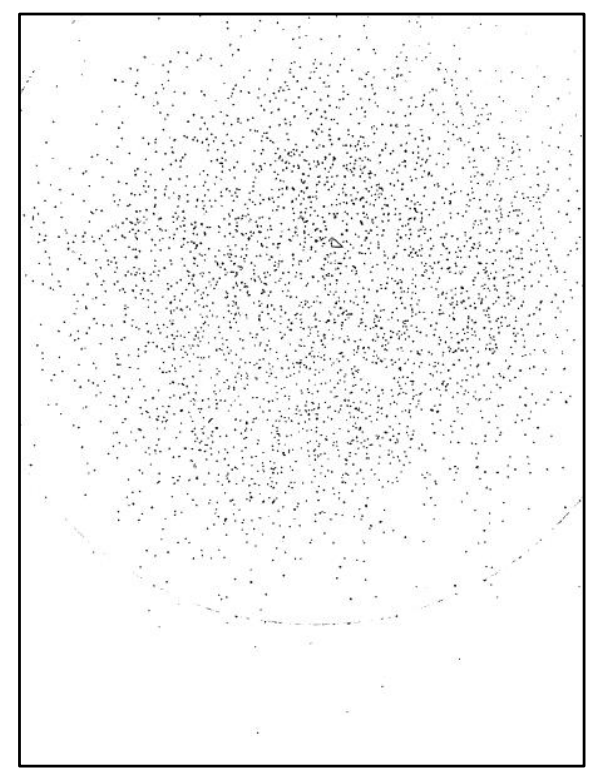

Figure 3. Picture of the results of the Gray-Level technique

\subsubsection{Segmentation and Detection Technique}

\section{Segmentation Technique}

The treatment of image segmentation can be realized by using four different principles, threshold method, zone method, boundary method, and edge method [6]. In the very small sample size of larvae, the segmentation is done by using a combination of the four methods to get good results.

\section{Detection Technique}

The BLOB analysis algorithm is used to find and count objects, and to make measurements based on their characteristics [7]. The purpose of the analysis is to determine whether the results are obtained from operations that are accurate, logical and correct. Image processing software consists of complex algorithms that have pixel values as input. Image analysis software packages today include old and new technology. Most significant is the relationship between the old BLOB analysis method and the new detection technique. For image processing, BLOB is defined as the area of connected pixels. BLOB analysis is the identification and study of this region in images $[8,9]$. This algorithm distinguishes pixels by their value and places them in one of two categories. That is. foreground (usually a pixel that has a non-zero value) or background (a pixel with a zero value).

Because the blob is the touching pixel area, the analysis tool usually considers touching the foreground pixels as part of the same blob. As a result what is easily recognized by the human eye as several different but touching blobs can be interpreted by the software as one blob. Furthermore, any part of the blob that is in the background pixel state due to lighting or reflection is considered as background during the analysis [10].
The performance of the BLOB analysis operation depends on successful image segmentation, separating the good blobs from the background and from each other and eliminating everything else in the image that is not attractive. Segmentation involves binarization operations [11]. If simple segmentation is not possible due to poor lighting or a blob with the same gray level as the background, we must develop a segmentation algorithm that is suitable for a particular image. Images obtained may contain fake noise or blobs or holes that may be caused by noise or lighting. Such foreign BLOB can interfere with the results of the BLOB analysis [12]. If the image contains several unfamiliar blobs, we must pre-process the image before using it. Preprocessing refers to whatever steps are taken to clean the image before analysis and can include thresholds or filtering [13].

\section{Result And Discussion}

Image data of zoea-1 larvae samples were taken and processed using image processing techniques using mobile camera instrumentation of three different types. Where the zoea phase is a phase that is very vulnerable in the life cycle of crab larvae. Overall the use of image processing techniques provides satisfactory results. We can see in one example of larval detection in Figure 5, that crab larvae can be detected and counted well.

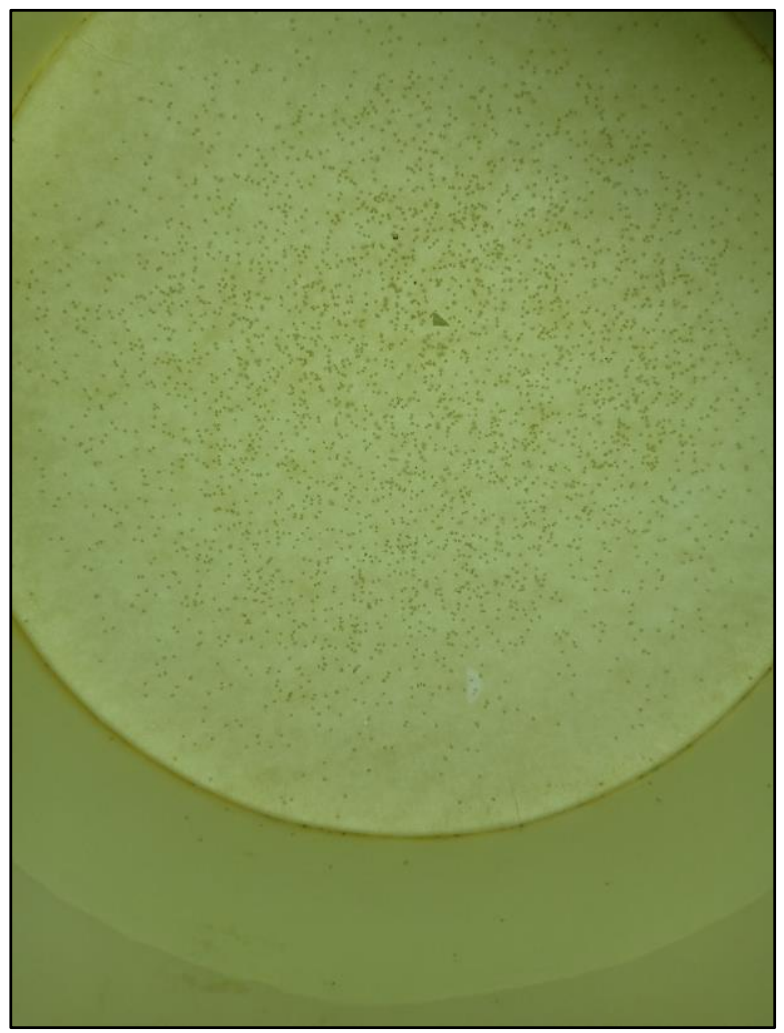

Figure 4. The results of taking pictures on the container 


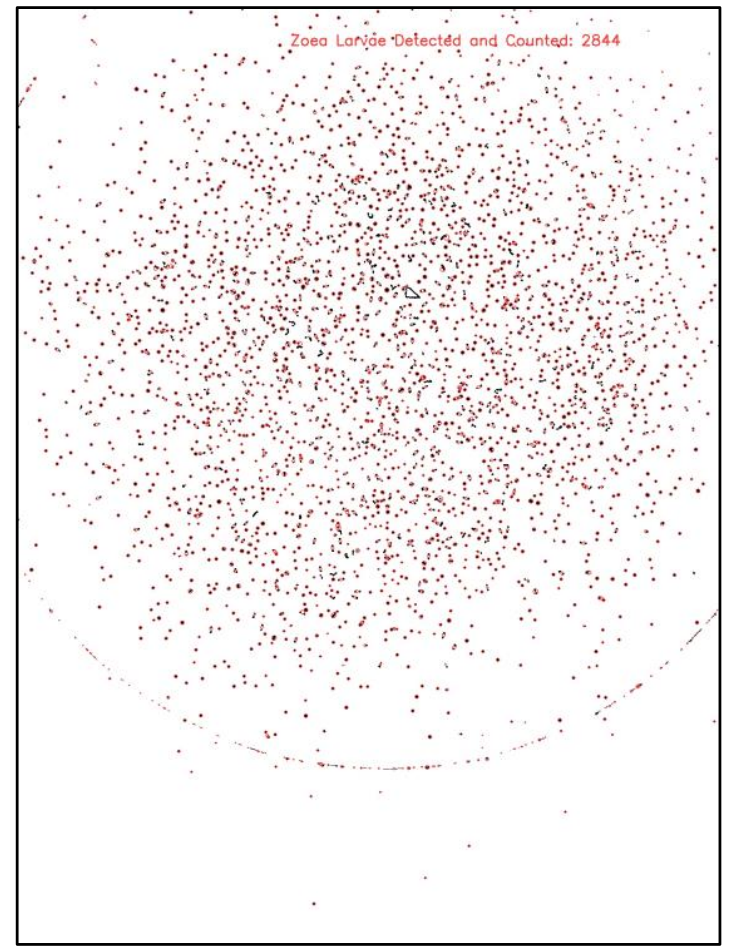

Figure 5. Detection and calculation results with counter application

In testing the system, the amount of image data on the Zoea-1 phase larvae is validated using the Confusion Matrix with the following accuracy testing results shown in the table.

$$
\text { Accuracy }=\frac{T P+T N}{T P+T N+F P+F N}
$$

where,

- $\mathrm{TP}$ is the number of larvae observations that are actually detected as larvae

- $\mathrm{TN}$ is the number of observations not larvae

- FP is the number of non-larvae detected as larvae

- FN is the number of larvae that cannot be detected

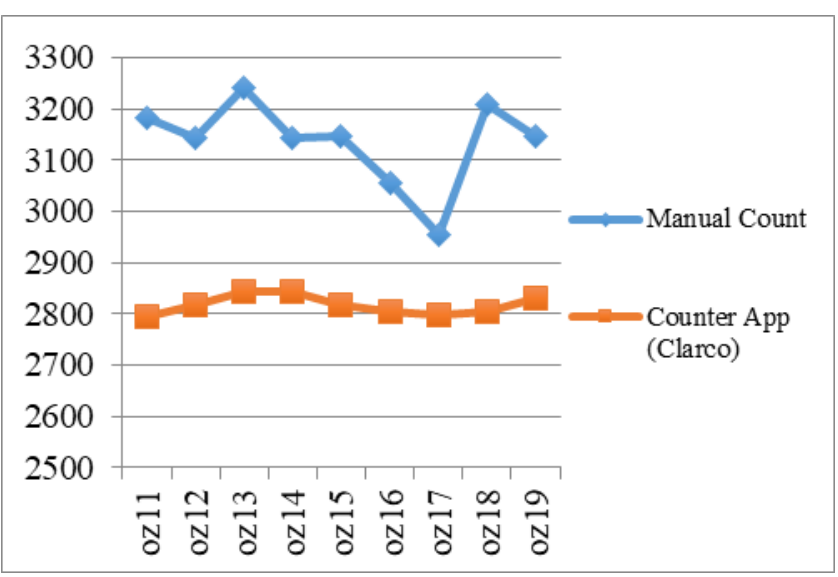

Figure 6. Comparison chart of counter application and manual calculations
Table 1. Zoea-1 system accuracy comparison

\begin{tabular}{cccc}
\hline Name & $\begin{array}{c}\text { Manual } \\
\text { Count }\end{array}$ & $\begin{array}{c}\text { Counter } \\
\text { App }\end{array}$ & $\begin{array}{c}\text { Accuracy } \\
\text { (TP+TN/TP+TN+FP+FN) \% }\end{array}$ \\
\hline oz11 & 3183 & 2794 & 87.78 \\
oz12 & 3145 & 2817 & 89.57 \\
oz13 & 3242 & 2844 & 87.72 \\
oz14 & 3144 & 2844 & 90.46 \\
oz15 & 3146 & 2817 & 89.54 \\
oz16 & 3055 & 2804 & 91.78 \\
oz17 & 2955 & 2799 & 94.72 \\
oz18 & 3210 & 2804 & 87.35 \\
oz19 & 3147 & 2832 & 89.99 \\
\multicolumn{2}{c}{ Average } & $\mathbf{8 9 . 8 8}$ \\
\hline
\end{tabular}

Table 2. Confusion matrix

\begin{tabular}{ccc}
\hline $\mathrm{n}=28227$ & $\begin{array}{c}\text { Prediction } \\
\text { True }\end{array}$ & $\begin{array}{c}\text { Prediction }= \\
\text { False }\end{array}$ \\
\hline Actual $=$ True & $\mathrm{TP}=25355$ & $\mathrm{FN}=2872$ \\
Actual $=$ False & $\mathrm{FP}=0$ & $\mathrm{TN}=0$ \\
\hline
\end{tabular}

$$
\begin{aligned}
\text { Accuracy } & =\frac{25355+T N}{28227} \\
& =0.898
\end{aligned}
$$

It can be seen from the table that the presentation accuracy of larvae detection results in the zoea-1 phase is $89.8 \%$. Containers containing defects, camera focus, reflected light, water level, and larval distance from the water surface are some of the things that affect the accuracy of larval detection.

\section{Conclusion and Future Work}

Calculation of the number of crab larvae in the zoea-1 phase using image processing techniques has a pretty good accuracy with an accuracy of $89.8 \%$. In this study, the image sample used was in the zoea- 1 phase and the image was taken in a special container with a limited volume of water so the larvae had to be removed from the hatchery tank. Taking pictures in various phases of zoea and direct calculation in the hatchery container and by using highlevel image recognition and intelligent algorithms is our next research in the future.

\section{Acknowledgment}

The author would like to thank C-BEST JICA for helping to fund this research. 


\section{References}

[1] A. Journal, V. Faj-, and P. In, "Survival Rate and Development Period of the Larvae of Survival Rate and Development Period of the Larvae of Portunus," vol. 2012, 2012.

[2] R. Nicholas and Z. Chaoshu, "Blue swimmer crabs: emerging species in Asia," Glob. Aquac. Advocate, vol. 11, no. 3, pp. 34-36, 2008.

[3] F. H. Ahmad, "From Pixels to Medical Imaging," ICSIPA 2013, p. 2013, 2013.

[4] A. Martin, S. Tosunoglu, and W. F. Street, "Image processing techniques for machine vision," pp. 1-9, 2000.

[5] M. Reynolds, "Image Processing Techniques," pp. 211-245, 2005.

[6] C. Yuchao and L. Hui, "Study on the Meso-Structure Image of Shale Based on the Digital Image Processing Technique," 2009.

[7] C. Damerval, S. Meignen, and A. O. Case, "Blob Detection With
Wavelet Maxima Lines," vol. 14, no. 1, pp. 39-42, 2007.

[8] P. Forss, "Robust Multi-scale Extraction of Blob Features," no. 2, pp. $11-18,2003$.

[9] G. Gerig, G. Szekely, G. Israel, and M. Berger, "Detection and Characterization of Detection and Characterization," June, pp. 165176, 1995.

[10] S. Hinz, "Fast and Subpixel Precise Blob Detection and Attribution Stefan Hinz Remote Sensing Technology."

[11] A. Ming, "A blob detector in color images," pp. 364-370, 2007.

[12] N. Khanina, E. Semeikina, and D. Yurin, "Color Blob and Ridge Detection," no. January 2010, 2014.

[13]A. Patil, M. E. Student, and M. Dhanvijay, "Blob Detection Technique using Image Processing for Identification of Machine," vol. 2, pp. 1-8, 2015. 